\title{
Stronger Functional Connectivity in the Default Mode and Salience Networks Is Associated With Youthful Memory in Superaging
}

\author{
Jiahe Zhang ${ }^{1}$, Joseph M. Andreano ${ }^{2,3}$, Bradford C. Dickerson ${ }^{3,4,5, *}$, \\ Alexandra Touroutoglou ${ }^{3,4,5, *}$ and Lisa Feldman Barrett ${ }^{1,2,3, *}$
}

${ }^{1}$ Department of Psychology, Northeastern University, Boston, MA 02115, USA, ${ }^{2}$ Psychiatric Neuroimaging Division, Department of Psychiatry, Massachusetts General Hospital and Harvard Medical School, Charlestown, MA 02129, USA, ${ }^{3}$ Athinoula A. Martinos Center for Biomedical Imaging, Massachusetts General Hospital and Harvard Medical School, Charlestown, MA 02129, USA, ${ }^{4}$ Department of Neurology, Massachusetts General Hospital and Harvard Medical School, Charlestown, MA 02129 and ${ }^{5}$ Frontotemporal Disorders Unit, Department of Neurology, Massachusetts General Hospital and Harvard Medical School, Charlestown, MA 02129, USA

Address correspondence to Bradford C. Dickerson, Frontotemporal Disorders Unit, 149 13th St, Suite 2691, Charlestown, MA 02129, USA. Email: brad.dickerson@mgh.harvard.edu; Alexandra Touroutoglou, Department of Neurology, Massachusetts General Hospital, 149 13th St, Suite 10.018, Charlestown, MA 02129, USA. Email: atouroutoglou@mgh.harvard.edu; Lisa Feldman Barrett, Department of Psychology, Northeastern University, 125 Nightingale Hall, Boston, MA 02115-5000, USA. Email: 1.barrett@northeastern.edu

*Made equivalent contributions and share senior authorship.

\begin{abstract}
"Superagers" are older adults who, despite their advanced age, maintain youthful memory. Previous morphometry studies revealed multiple default mode network (DMN) and salience network (SN) regions whose cortical thickness is greater in superagers and correlates with memory performance. In this study, we examined the intrinsic functional connectivity within DMN and SN in 41 young ( $24.5 \pm 3.6$ years old) and 40 older adults ( $66.9 \pm 5.5$ years old). Superaging was defined as youthful performance on a memory recall task, the California Verbal Learning Test (CVLT). Participants underwent a resting-state functional magnetic resonance imaging (fMRI) scan and performed a separate visual-verbal recognition memory task. As predicted, within both DMN and SN, superagers had stronger connectivity compared with typical older adults and similar connectivity compared with young adults. Superagers also performed similarly to young adults and better than typical older adults on the recognition task, demonstrating youthful episodic memory that generalized across memory tasks. Stronger connectivity within each network independently predicted better performance on both the CVLT and recognition task in older adults. Variation in intrinsic connectivity explained unique variance in memory performance, above and beyond youthful neuroanatomy. These results extend our understanding of the neural basis of superaging as a model of successful aging.
\end{abstract}

Key words: episodic memory, intrinsic functional connectivity, resting state network, successful aging 


\section{Introduction}

Episodic memory decline usually accompanies normal human aging (Grady and Craik 2000; Dixon et al. 2012). However, recent research has identified a specific subgroup of older adults, called "superagers," whose recall memory on tests such as the Rey Auditory Verbal Learning Test (RAVLT) or California Verbal Learning Test (CVLT) rivals that of middle-aged adults (Harrison et al. 2012; Rogalski et al. 2013; Gefen et al. 2014; Gefen et al. 2015) and even young adults (Sun et al. 2016; Dang et al. 2018; Harrison et al. 2018). More importantly, superagers are characterized by youthful neuroanatomy in a consistent ensemble of brain regions within the topography of 2 large-scale brain networks (Fig. S1; Sun et al. 2016; Harrison et al. 2018)—the default mode network (DMN; Raichle et al. 2001; Greicius et al. 2003) and the salience network (SN; Seeley et al. 2007; Touroutoglou et al. 2012). These regions typically show age-related atrophy in healthy older adults (McGinnis et al. 2011; Bakkour et al. 2013; Crivello et al. 2014; Fjell et al. 2014).

Within the DMN, the hippocampal formation (HF) (Squire et al. 2004), medial temporal cortices, posterior cingulate cortex (PCC), and adjacent midline parietal areas (Dickerson and Eichenbaum 2009) have all been implicated in successful memory encoding and retrieval. Within the $\mathrm{SN}$, the anterior insula, anterior to midcingulate cortex (MCC), middle frontal gyrus, and inferior parietal lobule support episodic memory encoding and retrieval (Wiggs et al. 1999; Lepage et al. 2000; Kim 2010; Sestieri et al. 2014), possibly by directing attention to relevant material, engaging working memory, organizing ("controlling") available information strategy, and adjusting motivation (Menon and Uddin 2010; Menon 2015). In older adults, age-related atrophy in a number of DMN and SN regions is associated with memory decline (e.g., Brickman et al. 2007; Kramer et al. 2007; Head et al. 2008), whereas youthful gray matter integrity predicts youthful verbal memory performance (Sun et al. 2016).

Gray matter atrophy (i.e., neuronal death) leads to disappearance or rewiring of synapses, which affects anatomical as well as functional connections between the atrophied region and other regions within its network ("network degeneration hypothesis"; Buckner et al. 2005; Palop et al. 2006; Seeley et al. 2009; Greicius and Kimmel 2012).Consistent with this hypothesis, typical aging is associated with progressively weaker intrinsic functional connectivity in the DMN (e.g., Andrews-Hanna et al. 2007; Esposito et al. 2008; Grady et al. 2012; Wang et al. 2012; Salami et al. 2014; Ward et al. 2015) and SN (e.g., Allen et al. 2011; Onoda et al. 2012; Wang et al. 2012; He et al. 2013; Roski et al. 2013; He et al. 2014; Langner et al. 2015; Touroutoglou et al. 2018). Weaker intrinsic connectivity between regions within the DMN has been linked to worse memory performance both in young (Touroutoglou et al. 2015) and in older adults (Andrews-Hanna et al. 2007; Wang et al. 2010; Ward et al. 2015). Weaker connectivity between regions within the $\mathrm{SN}$ has been associated with worse memory performance in young adults (Andreano et al. 2017).

In the current study, we built on these existing structural imaging findings to perform the first-to our knowledgeinvestigation of the functional integrity of the DMN and SN in superaging. We examined intrinsic functional connectivity of the DMN and SN in the same sample of healthy older and young adults as discussed in the study by Sun et al. (2016). Although prior studies have demonstrated superior structural integrity at advanced ages of 80 and above (Harrison et al. 2012; Rogalski et al. 2013; Gefen et al. 2014; Gefen et al. 2015), their design did not allow for brain characteristics to be observed during the time period when cognitive decline generally accelerates. To study this period, we sampled a younger age range (i.e., 60-80; see reviews in Hedden and Gabrieli 2004; Park and Reuter-Lorenz 2009; Nyberg et al. 2012) and accordingly, used young adults ages $18-35$ as benchmark instead of middleaged adults. We hypothesized that superagers (i.e., individuals with CVLT scores comparable to young adults) would have stronger intrinsic connectivity within the DMN and SN relative to typical older adults, similar to that observed in young adults. We additionally examined their visual-verbal recognition memory on an independent memory test and hypothesized that superagers would show youthful memory performance on both item and associative recognition memory, demonstrating that their superior memory function generalized beyond their performance on the CVLT. Finally, we hypothesized that within older adults, individuals with stronger intrinsic DMN and SN connectivity would perform better on both the verbal recall task (the CVLT) and the visual-verbal recognition task (for both item and associative memory). Given the different functional roles of the DMN and SN in memory, we hypothesized that DMN and SN connectivity would each independently predict memory performance relative to one another, as well as above and beyond the variance explained by the anatomical integrity of regions in those networks.

\section{Materials and Methods}

\section{Participants and Study Procedure}

Ninety-one participants (48 males) were initially recruited from the Greater Boston area, comprising 47 young adults (24 males, ages $18-35$ ) and 44 older adults (24 males, ages 60-80). All participants were right-handed native English speakers with normal or corrected-to-normal vision and none reported a history of substance neurological or psychiatric disorder. All experiments involving human subjects were approved by the Massachusetts General Hospital Institutional Review Board. For all neuropsychological instruments used, participants were required to score within 1.5 standard deviations of published normative values based on their age and education. Ten participants were disqualified due to incomplete study procedure, resulting in a final sample size of 41 young adults (20 males, $24.5 \pm 3.6$ years) and 40 older adults (17 males, $66.9 \pm 5.5$ years), same as discussed in the study by Sun et al. (2016). These participants were part of a larger, longitudinal study, allowing us to report additional data and analyses that were not yet available at the time the initial report was submitted for publication.

Participants arrived at the lab on day 1 to complete a neuropsychological battery of tests, including the CVLT (Delis et al. 1987) and the Trail Making Test (TMT) (Tombaugh 2004). On day 2 ( 1 to 3 days later), participants underwent structural and resting-state scans. On day 3 (another 1 week later), participants completed an associative memory task (Andreano et al. 2017). Paired associates consisted of 120 face-word pairs and 120 scene-word pairs. All stimuli were chosen carefully to be affectively neutral. Face stimuli were obtained from the Center for Vital Longevity Face Database (Minear and Park 2004) and depicted male and female faces from multiple age groups. Scene stimuli were obtained from the International Affective Picture System (Lang et al. 1997) and were neutral in valence and arousal. Words were selected from the Medical Research Council Psycholinguistic Database (Coltheart 1981). All words 
were adjectives, selected for high frequency and high concreteness. Each image/word pair was presented for $6 \mathrm{~s}$, and a total of 20 pairs (10 scene/word and 10 face/word) were encoded in a single run and a total of 4 runs were conducted. To ensure depth of encoding, participants were asked to judge whether the word "matched" the picture. As picture/word pairs were created randomly, and pairs with an obvious semantic connection were excluded, this judgment was subjective. After a 10-min retention delay, participants were presented with all 80 pairs learned during encoding, as well as 40 pairs made up of new words and pictures, and 40 rearranged pairs made of words and pictures seen previously, but not previously associated. Each picture was presented for $6 \mathrm{~s}$, during which time the participant responded by button press whether the pair had appeared during encoding, or whether it was a new or rearranged pair (yes/no). Each recognition trial was coded as a hit, miss, false alarm, or correct rejection, and recognition accuracy was computed in terms of d', a measure that controls for individual response bias [d' $=z$ (hits) $-z$ (FA)]. We calculated d' separately for item recognition (previously encoded pairs vs. novel pairs) and associative recognition (previously encoded pairs vs. rearranged pairs). Two participants were excluded from CVLT-related analyses due to missing data. One participant was excluded from recognition-related analyses due to being an outlier on item recognition score. Demographic information, memory task data, group level connectivity maps, t-test maps, network masks, and connectivity strength data can be retrieved on Open Science Framework (doi:10.17605/OSF.IO/G6F2K; ark:c7605/osf.io/g6f2k). Raw data and analysis scripts are also available upon request.

\section{Superaging Definition}

As in our previous study (Sun et al. 2016), we defined superagers ( $n=17,5$ males, $67.8 \pm 6.0$ years) as those who performed at or above the mean for young adults (ages 18-32) on the long delay free recall measure of the CVLT (males: 13; females: 14) and no lower than 1 standard deviation below the mean for their age group on the TMT Part B. There are well-established gender differences in episodic memory throughout the life span (Herlitz et al. 1997; Herlitz and Rehnman 2008), and standard tests are often normed according to gender for more accurate reflection of the populations. Therefore, using gender-adjusted means allows us to compare each older adult's memory capacity to their respective, gender-appropriate youthful baseline and avoid gender-related confounds in cognitive aging.

\section{MRI and AMRI}

Imaging data were acquired in a three Tesla Magnetom Tim Trio system (Siemens Medical Systems, Iselin, NJ). Structural data were acquired using a T1-weighted MPRAGE sequence (TR/TE/FA $=2530 \mathrm{~ms} / 3.48 \mathrm{~ms} / 7$ degrees; slice thickness $=1 \mathrm{~mm}$; FoV $=256 \mathrm{~mm}$; 0\% slice gap). We reconstructed the cortical surface using the automated algorithm in FreeSurfer (http:// surfer.nmr.mgh.harvard.edu) and extracted cortical thickness/ subcortical volume from superaging brain regions as discussed in the study by Sun et al. (2016). Resting-state data were acquired using an echo-planar sequence (TR/TE/FA $=5000 \mathrm{~ms} / 30 \mathrm{~ms} / 90$ degrees; slice thickness $=2 \mathrm{~mm}$; matrix size $=256 \mathrm{~mm}$; $0 \%$ slice gap; $6.40 \mathrm{~min}, 76.8$ volumes). To preprocess the restingstate data, we removed first 4 volumes, corrected slice timing (SPM2), corrected head motion using the first volume as reference (FSL), normalized to the MNI152 template (SPM2), resampled to 2-mm cubic voxels, removed frequencies higher than $0.08 \mathrm{~Hz}$, smoothed with a $6-\mathrm{mm}$ fwhm kernel, and did nuisance regression (6 motion parameters, average global signal, average ventricular, and white matter signals) (Biswal et al. 1995; Vincent et al. 2007; van Dijk et al. 2010). Consistent with earlier publications based on this set of resting-state data (Andreano et al. 2017; Touroutoglou et al. 2018), we performed motion denoising using 6 motion parameters and global signal. Recent evidence indicated that adding first derivatives and down-weighting outlier volumes minimized motion-related confounds in group comparisons (Ciric et al. 2017). Our current results are likely unaffected by motionrelated confounds since superagers and typical older adults did not differ in mean displacement $[t(38)=-1.55, P=0.13$; 2-tailed] or number of spikes [ $t(38)=-0.81, P=0.42]$, calculated following procedures outlined in (Van Dijk et al. 2012). When not specified, preprocessing steps were implemented using a custom script used in previous reports (Bickart et al. 2012; Touroutoglou et al. 2012; Touroutoglou et al. 2014; Touroutoglou et al. 2015; Andreano et al. 2017).

\section{Within-Network Functional Connectivity and Behavioral Correlation Analyses}

A priori DMN and SN masks were defined in an independent sample of 89 young adults (44 men; aged 22.4 \pm 3.34 ) (Fig. S1; Touroutoglou et al. 2012; Sun et al. 2016). For details on mask creation, see the study by Sun et al. (2016). To explore the topography of functional connectivity in our aging sample, we generated 4-mm spherical regions of interest (ROIs) in the left PCC (MNI 1, -55, 17) (Vincent et al. 2008) to identify the DMN and in the right dorsal anterior insula (dAI; MNI 36, 21, 1) (Touroutoglou et al. 2012) to identify the SN. We obtained Pearson's product moment correlations, $r$, between the average time course within each seed ROI and that of all voxels across the brain, converted those $r$ values to $z$ values using Fisher's r-to$z$-transformation, and averaged the resulting $z$ maps across all subjects to obtain a group map per network. We projected the group maps onto the FreeSurfer fsaverage surface (Fig. S2A). For each network, we conducted a voxel-wise 2-sample ttest between superagers and typical older adults, masked the resulting map by its corresponding a priori network mask, and thresholded it at $\mathrm{P}<0.05$ (uncorrected; Fig. S2B). To identify network targets where superagers had stronger connectivity than typical older adults, we followed a standard procedure as previously published by our lab (Touroutoglou et al. 2012; Touroutoglou et al. 2016): for each gray matter cluster on the masked contrast maps, we identified the voxel with the highest $z$ value as the peak, and these were used as targets (list of targets with peak MNI coordinates can be found in Table S1). To quantify the strength of functional connectivity between seed and target regions, for all subjects in the older adult group, we created spherical ROIs $(4 \mathrm{~mm}$ ) around each correlation peak and extracted the averaged time course within each ROI. We next calculated Pearson's $r$ between seed and all target time courses and applied Fisher's $r$-to- $z$ transformation. This resulted in 1 connectivity strength score per network target per subject. We conducted 2-tailed planned contrasts on connectivity strength 1 ) between superagers and young adults to test whether superagers showed youthful levels of network coupling and 2) between superagers and typical older adults to confirm superagers had tighter network coupling than normal older adults. Finally, we calculated Pearson's $r$ between each 
network target's connectivity strength and 1) recall memory scores to assess concurrent criterion validity as well as 2) item and associative recognition memory scores to assess construct validity. To correct for multiple comparisons across 15 bivariate correlations for each memory test, we tested the $P$ values for significance using false discovery rate (FDR) $q$ of 0.05 following the Benjamini-Hochberg procedure (Benjamini and Hochberg 1995). To show specific contribution of the DMN and SN, we selected 2 additional networks as control networks. Motor network seed was located in the left primary motor cortex (M1: MNI -43, $-16,42)$ as in Andoh et al. (2015), and visual network seed was located in the primary visual cortex (V1: MNI $-19,-98,-3)$ as in Konishi et al. (2000). We followed the same procedure as DMN and SN to identify motor and visual network targets (Table S1) that showed stronger connectivity in superagers than typical older adults, and calculated connectivity strength between each control network seed and target for all subjects in the older adult group. Finally, we also calculated Pearson's $r$ between control network connectivity strength and the 3 memory task scores.

\section{Post-Hoc Between-Network Functional Connectivity and Behavioral Correlation Analyses}

To examine connectivity difference across networks, we masked the PCC-seeded t-test map by the SN mask and masked the dAIseeded t-test map by the DMN mask. We observed a number of regions that showed between-network connectivity differences in superagers. Given the importance of the MCC in superaging (Harrison et al. 2012; Rogalski et al. 2013; Gefen et al. 2014; Sun et al. 2016), we investigated the connectivity between the bilateral MCC and the PCC seed. We located a voxel with the highest $\mathrm{z}$ value within each MCC peak (L MCC: $-2,10,48 ; \mathrm{R}$ MCC: $8,14,48$ ) and calculated Pearson's $r$ between its time course and the PCC time course for each subject. We conducted 2-tailed planned contrasts on connectivity strength between young adults, superagers, and typical older adults. We also calculated Pearson's $r$ between connectivity strength and the 3 memory task scores (recall, item recognition, and associative recognition).

\section{Brain-Behavior Regression Analysis}

We ran 1 hierarchical linear regression analysis for each index of memory as the dependent measure, using intrinsic connectivity strength between canonical network nodes as predictor variables. We picked key nodes within the DMN and SN and used their connectivity strength as predictors in each regression model. For the DMN, the predictor was PCC connectivity to the right $\mathrm{HF}$; for the $\mathrm{SN}$, the predictor was dAI connectivity to the left MCC connectivity. We conducted 2 regression analyses, one using recall as the outcome measure and the other using item recognition as outcome measure. We did not conduct an analysis for associative recognition since it was not significantly predicted by connectivity between the dAI seed and any SN target. Further, given that the study by Sun et al. (2016) showed greater cortical thickness in older adults predicted memory, we examined the contributions of both neuroanatomy and connectivity memory. Specifically, we ran regression analyses using neuroanatomy and functional connectivity as predictors for recall memory (CVLT). To index anatomical integrity, we initially planned to include cortical thickness/volume estimates for the regions used to compute estimates of intrinsic connectivity (i.e., R HF and R PCC for the DMN, L MCC and R dAI for the SN). R PCC thickness was not greater in superagers (Sun et al. 2016) and R dAI thickness was not independently associated with CVLT performance over and above $\mathrm{R} \mathrm{HF}$ volume and $\mathrm{L}$ MCC thickness, and so we excluded R PCC and R dAI from the analyses. As a consequence, we ran 2 regression analyses to predict CVLT performance, one using R HF volume and R HF$\mathrm{R}$ PCC connectivity as DMN predictors, and the other using L MCC thickness and L MCC-R dAI connectivity as SN predictors, respectively. The structural data were calculated based on the HF and MCC labels from (Sun et al. 2016). Then we ran 1 additional regression analysis with anatomical and functional variables of both networks as predictors. Cortical thickness measures did not significantly predict item recognition memory or associative recognition memory so additional regression analyses were not necessary in that regard. For all above tested hierarchical regression models, we further added L MCC-R PCC connectivity strength as an index for between-network connectivity to test whether it uniquely contributed to memory. As a complementary analysis to this key node approach, we also conducted a principal component analysis on all 17 seed-target connectivity pairs within DMN, with SN, as well as between DMN and SN, and found 2 components representing the DMN and SN (see Text S1 and Fig. S3 in Supplementary Material). Factor scores derived from these components were entered into a regression model together with both DMN and SN structural integrity estimates. Lastly, we verified that functional connectivity estimates significantly explained unique variance 1) when we extracted cortical thickness values from the ROIs used in this paper and 2) when we controlled for the cortical thickness of seed and target regions that did not differ in thickness between superagers and typical older adults, as indicated by Sun et al. (2016) (see Text S2 in Supplementary Material).

\section{Results}

\section{Default Mode and SN Intrinsic Connectivity Is Stronger in Superagers}

The average intrinsic functional connectivity map for superagers and typical older adults can be found in Figure S2 (for cortical regions) and Figure $3 \mathrm{~A}$ (for the hippocampus). The map for the DMN was anchored in a PCC seed as used in the study by Vincent et al. (2008); the map for the SN was anchored in a seed within dorsal anterior insula, or $\mathrm{dAI}$ as used in (Touroutoglou et al. 2012).

For each network, a 2-sample t-test between superagers and typical older adults revealed that superagers had stronger intrinsic connectivity between the network seed and its targets (Fig. 1A; Fig. 3B). Within the DMN, superagers showed stronger connectivity from the right PCC seed to right HF (Fig. 3B) as well as to a number of cortical targets, such as left superior frontal gyrus (SFG), right anterior middle temporal gyrus (aMTG), right ventrolateral prefrontal cortex (vlPFC), right pregenual anterior cingulate cortex (pgACC), and right subgenual anterior cingulate cortex (sgACC) as well as bilateral angular gyrus (AG), dorsomedial prefrontal cortex (dmPFC), and rostromedial prefrontal cortex (rmPFC) (Fig. 1A). Within the SN, superagers showed stronger intrinsic connectivity from the right dAI seed to the right supramarginal gyrus (SMG) and bilateral anterior MCC (Fig. 1A). Furthermore, 2-tailed planned contrast tests verified that superagers had significantly stronger connectivity when compared with typical older adults $(P<0.05$; except left 


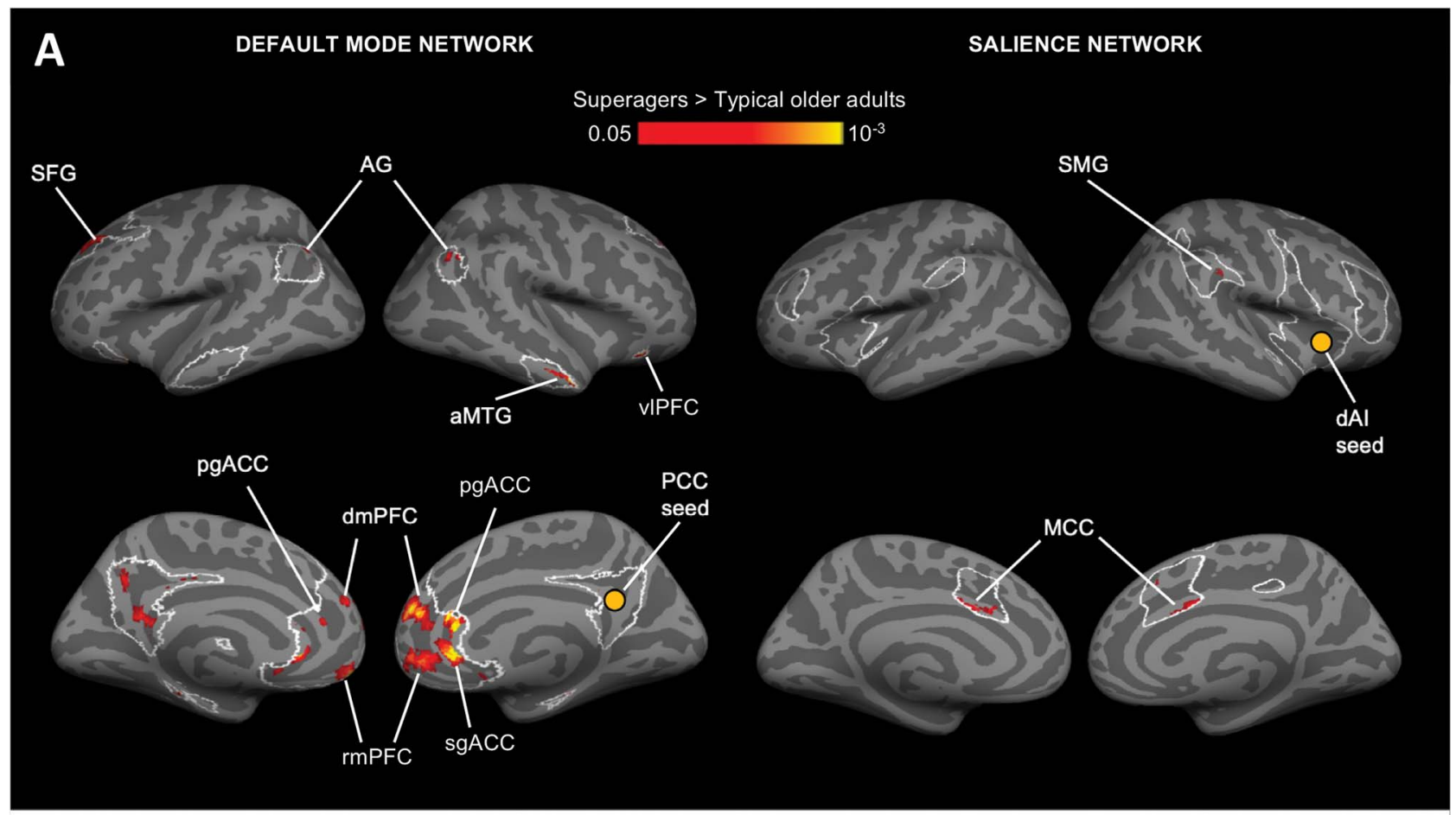

B

DEFAULT MODE NETWORK

SALIENCE NETWORK

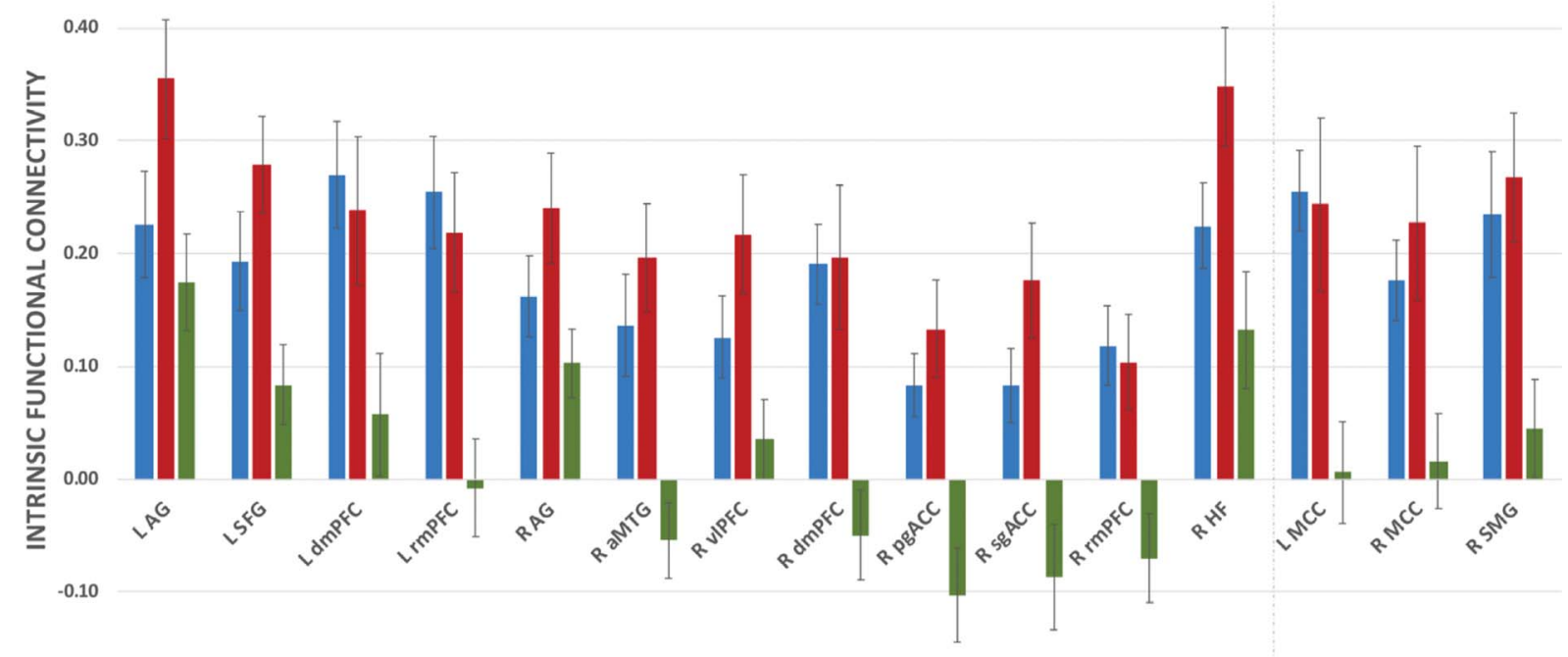

Q Young adults $\quad$ Superagers Typical older adults

Figure 1. Stronger DMN and SN connectivity in superagers. (A) Regions of the DMN and SN (outlined in white) where superagers had stronger intrinsic functional connectivity than did typical older adults (red/yellow). For each network, a 2-sample t-test between superagers and typical older adults was conducted. Maps were thresholded at $P<0.05$ and masked by network masks shown in Figure S1. We did not observe any region with higher connectivity in typical older adults than in superagers. We excluded clusters in the left precuneus/PCC region from further analyses because they showed high auto-correlation. (B) Bar graphs show superagers had stronger intrinsic connectivity within DMN and SN than did typical older adults $(P<0.05)$ but similar connectivity to young adults $(P>0.05)$. We calculated intrinsic connectivity strength between each network seed and its targets identified from peaks in the t-test maps in Figure 3. R, right hemisphere; L, left hemisphere. Error bars indicate 1 standard error of the mean. In several comparisons, the bars for superagers (orange) were higher than those for young adults (blue) and the error bars did not overlap. However, statistically, superagers were indistinguishable from young adults in all comparisons. 


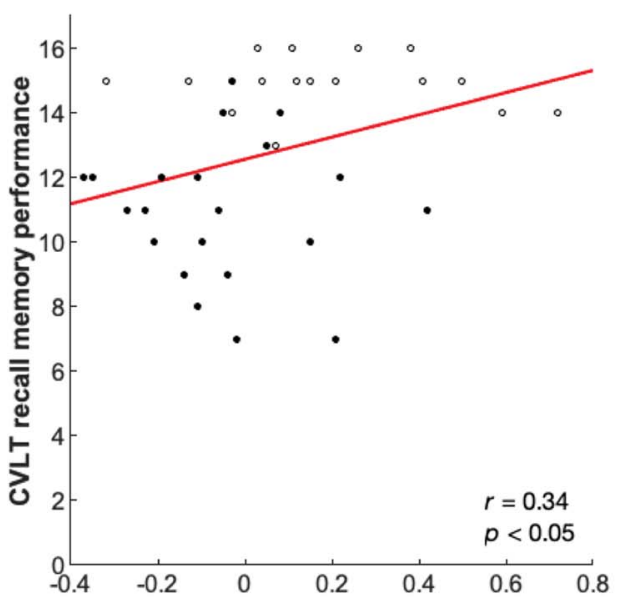

R dmPFC-R PCC intrinsic functional connectivity

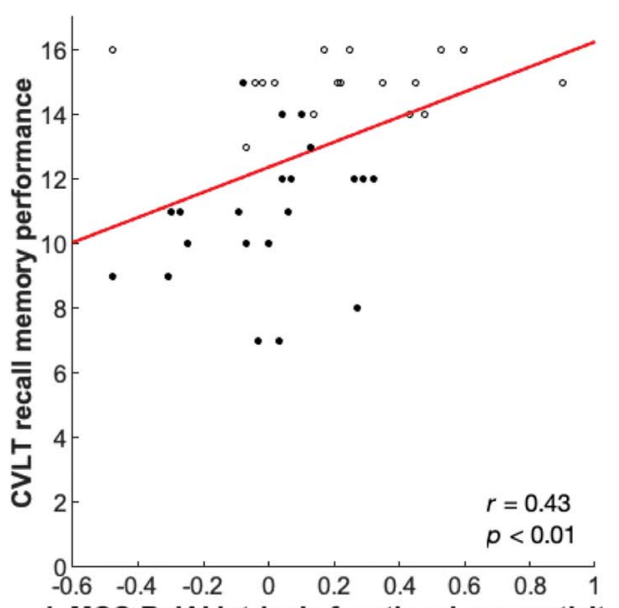

L MCC-R dAl intrinsic functional connectivity

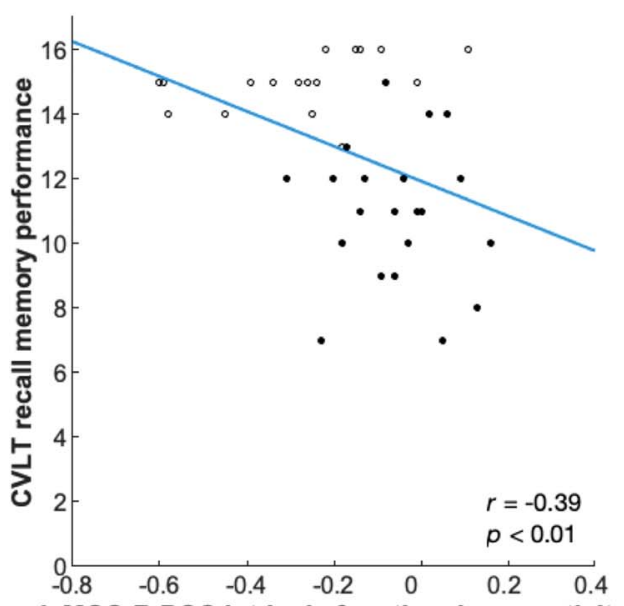

L MCC-R PCC intrinsic functional connectivity
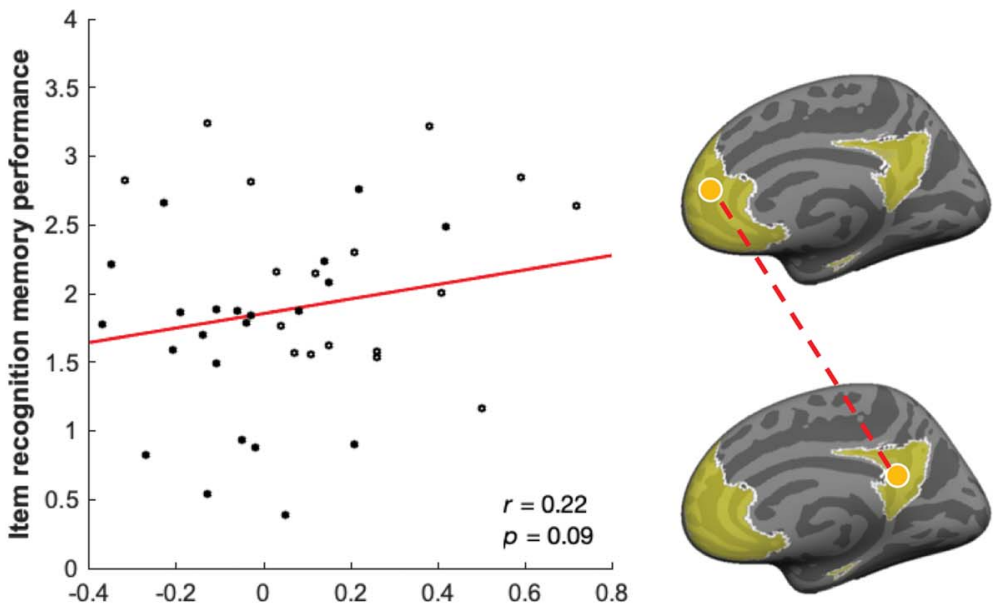

R dmPFC-R PCC intrinsic functional connectivity
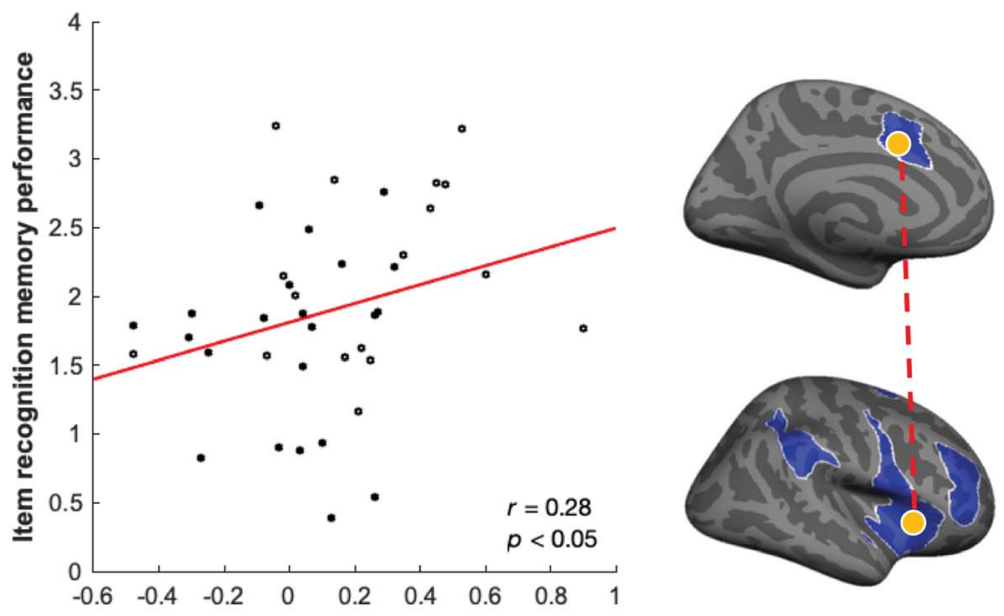

L MCC-R dAI intrinsic functional connectivity
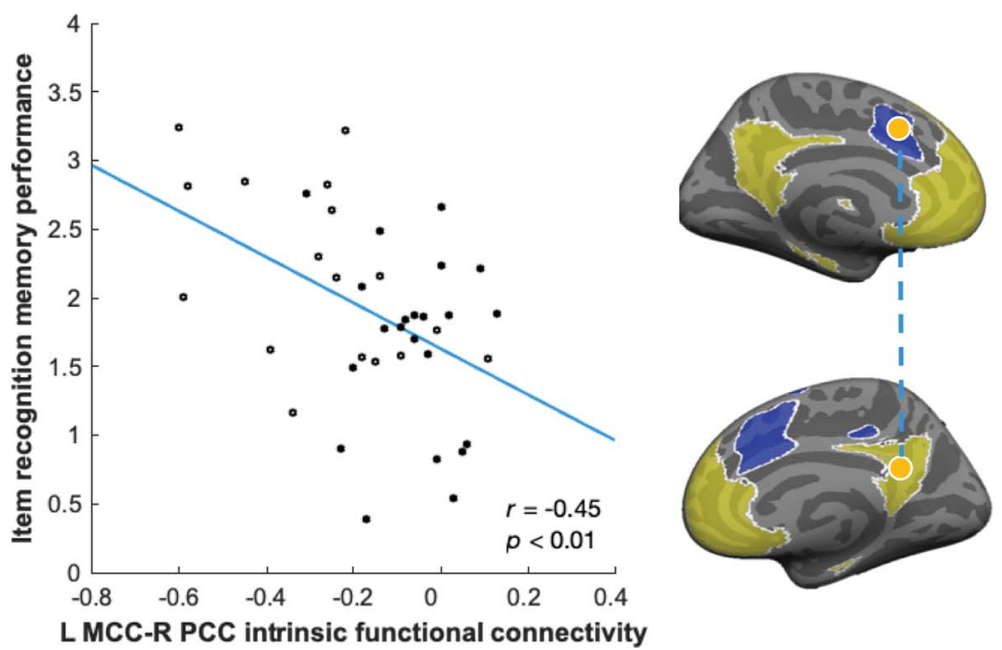

Figure 2. Stronger functional connectivity within and between default mode and SNs supports better memory in older adults. Scatterplots illustrate the correlation in the entire older adult group between intrinsic functional connectivity (top row: within DMN; middle row: within SN; bottom row: between DMN and SN) and memory performance (left column: CVLT recall for assessing criterion validity; central column: item recognition for assessing construct validity). Superagers are indicated by hollow points. The brain maps (right column) highlight the hypothesized networks of interest (DMN in yellow and SN in blue), seed and target nodes (in orange), and the direction of correlation representing functional connectivity (direct/positive in red and inverse/negative in light blue). Recall memory was scored out of a total of 16. Network seeds were R PCC (Vincent et al. 2008) and R dAI (Touroutoglou et al. 2012). Displayed P values are uncorrected. 
dmPFC, $P=0.05)$, whereas their intrinsic connectivity strength was statistically indistinguishable from young adults $(P>0.05$; Fig. 1B).

We also conducted a post-hoc analysis examining connectivity between the DMN and SN (Fig. S4A). We found that superagers and young adults demonstrated similar connectivity between the right PCC (DMN node) and bilateral MCC (SN node) $(P>0.05)$, and that the inverse (negative) connectivity in young adults and superagers was not seen in typical older adults $(P<0.05)$ (Fig. S4B).

\section{Youthful Recall and Recognition Memory Performance in Superaging}

We examined the generalizability of youthful memory in superagers using a visual-verbal paired-associates recognition memory task. Two 1-way analysis of variance showed that the 3 groups differed on their item recognition memory $[F(2,77)=$ $3.26, P=0.04]$ and associative recognition memory $[F(2,77)=4.41$, $P=0.02]$. Planned contrasts indicated, as predicted, that superagers performed better than typical older adults in item recognition $[t(77)=2.04, P=0.02$, 1-tailed] and marginally better in associative recognition $[t(77)=1.44, P=0.08,1$-tailed] but did not differ significantly from young adults [item recognition: $t(77)=-0.11, P=0.92$, 2-tailed; associative recognition: $t(77)=1.10$, $P=0.27$, 2-tailed]. To estimate the effect size of the difference between superagers and typical older adults, we computed Cohen's $d$ for each measure. Item recognition showed a large difference $[d=0.80,95 \%$ confidence interval $(C I)=0.49-1.08]$, while associative recognition showed a moderate difference $(d=0.48,95 \% \mathrm{CI}=0.08-0.72)$. For comparison, CVLT free recallused to define the groups-showed a large difference: $d=2.37$, $95 \% \mathrm{CI}=1.96-3.26$.

\section{Intrinsic Connectivity Predicted Recall and Recognition Memory Performance}

We ran a series of bivariate correlation analyses between all 3 memory measures (CVLT verbal recall, visual-verbal item recognition, and visual-verbal associative recognition) and connectivity strengths of all 15 seed-target pairs (including both DMN and SN). As predicted, intrinsic connectivity strength of all seedtarget pairs predicted recall performance in older adults at a FDR (q) of 0.05 (Table 1; scatterplots for key nodes are depicted in left column of Fig. 2 and Fig. 3C).

Importantly, this brain-behavior relationship generalized to item recognition memory as well (Table 1; scatterplots for key nodes are depicted in central column of Fig. 2). Item recognition memory was significantly correlated with multiple indices of DMN connectivity strength (FDR-corrected at $q=0.05$; Table 1 ). Additionally, a trend-level relationship was observed between item recognition memory and all 3 indices of SN connectivity strength, as well as between associative recognition memory and DMN network strength (Table 1).

Post-hoc analysis of between-network connectivity revealed that connectivity (stronger inverse correlation) between right PCC and left MCC predicted better performance on CVLT (Fig. 2C), item recognition, and associative recognition; connectivity between right PCC and right MCC predicted only CVLT (Table S2). Out of all 6 correlations tested between memory performance and control network connectivity strength, we only found motor network connectivity to be positively associated with CVLT (Table S3).

To test whether DMN and SN intrinsic connectivity each explained unique variance in memory performance, we ran 1 hierarchical linear regression analysis for each index of memory as the dependent measure, using intrinsic connectivity strength between canonical network nodes as predictor variables. We
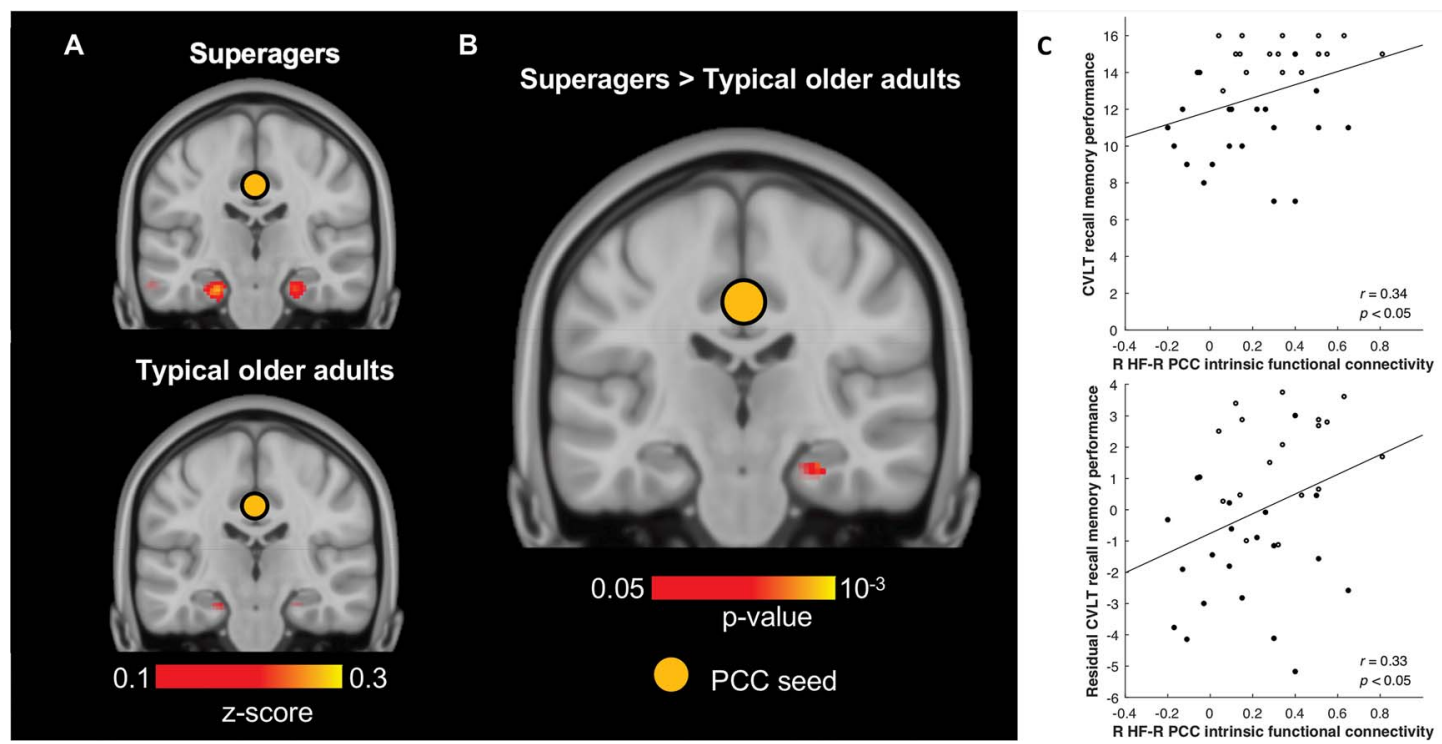

Figure 3. Stronger functional connectivity between hippocampus-a key node in the DMN-and PCC correlates with better memory in older adults. (A) Hippocampal connectivity to PCC seed (indicated by orange circle) in both older adult groups $(z>0.1)$. (B) 2-sample t-test revealed that superagers had significantly stronger $\mathrm{R}$ HF- $R$ PCC connectivity than typical older adults $(P<0.05)$. Hippocampal slices in both $A$ and $B$ were taken at $y=-22$ and displayed in neurological convention. (C) R HF-R PCC connectivity positively predicted raw CVLT recall memory performance (top) and its residual after controlling for right hippocampal volume (bottom) in the entire older adult group. 
Table 1 Association between memory and intrinsic connectivity "within" DMN and SN

\begin{tabular}{|c|c|c|c|c|c|c|c|}
\hline \multirow{2}{*}{\multicolumn{2}{|c|}{ Target region }} & \multicolumn{2}{|c|}{ Recall } & \multicolumn{2}{|c|}{ Item recognition } & \multicolumn{2}{|c|}{ Associative recognition } \\
\hline & & \multirow[t]{2}{*}{$r$} & \multirow[t]{2}{*}{$p$} & \multirow[t]{2}{*}{$r$} & \multirow[t]{2}{*}{$p$} & \multirow[t]{2}{*}{$r$} & \multirow[t]{2}{*}{$p$} \\
\hline & & & & & & & \\
\hline & L AG & 0.44 & $0.00^{*}$ & 0.17 & 0.15 & 0.23 & 0.08 \\
\hline & L SFG & 0.34 & $0.02 *$ & -0.03 & 0.43 & 0.07 & 0.33 \\
\hline & $\mathrm{L}$ dmPFC & 0.34 & $0.02^{*}$ & 0.22 & 0.09 & 0.37 & $0.01^{\dagger}$ \\
\hline & L rmPFC & 0.46 & $0.00^{*}$ & 0.12 & 0.23 & 0.29 & 0.04 \\
\hline & R AG & 0.39 & $0.01^{*}$ & 0.49 & $0.00^{*}$ & 0.36 & $0.01^{\dagger}$ \\
\hline & R aMTG & 0.47 & $0.00^{*}$ & 0.41 & $0.01^{*}$ & 0.32 & $0.02^{\dagger}$ \\
\hline & R vlPFC & 0.40 & $0.01^{*}$ & 0.43 & $0.00^{*}$ & 0.23 & 0.08 \\
\hline & $\mathrm{R}$ dmPFC & 0.34 & $0.02^{*}$ & 0.20 & 0.12 & 0.12 & 0.24 \\
\hline & R pgACC & 0.41 & $0.01^{*}$ & 0.30 & $0.03^{\dagger}$ & 0.19 & 0.13 \\
\hline & R sgACC & 0.32 & $0.03^{*}$ & 0.26 & $0.06^{\dagger}$ & 0.16 & 0.17 \\
\hline & R rmPFC & 0.38 & $0.01^{*}$ & 0.27 & $0.05^{\dagger}$ & 0.12 & 0.23 \\
\hline & R HF & 0.34 & $0.02^{*}$ & 0.27 & $0.05^{\dagger}$ & 0.32 & $0.02^{\dagger}$ \\
\hline \multicolumn{8}{|c|}{ SN } \\
\hline & L MCC & 0.43 & $0.00^{*}$ & 0.28 & $0.04^{\dagger}$ & 0.09 & 0.30 \\
\hline & R MCC & 0.39 & $0.01^{*}$ & 0.31 & $0.03^{\dagger}$ & 0.20 & 0.11 \\
\hline & R SMG & 0.38 & $0.01^{*}$ & 0.29 & $0.04^{\dagger}$ & 0.22 & 0.09 \\
\hline
\end{tabular}

Note: $r=$ Pearson's correlation coefficients, $P=1$-tailed significance. FDR-corrected significance: $* q<0.05,{ }^{\dagger} q<0.10$.

observed that stronger intrinsic DMN connectivity $(B=0.31$, $P=0.04)$ and stronger SN connectivity $(B=0.41, P=0.01)$ independently predicted better memory performance, accounting for a total of $28 \%$ of the variance in the CVLT recall score $[F(2,35)=6.89$, $P=0.00$; first data row of Table S4]. DMN $(B=0.26, P=0.10)$ and SN connectivity $(B=0.28, P=0.08)$ also explained $15 \%$ of the variance in item recognition score $[F(2,36)=3.14, P=0.06$; second data row of Table S4]. Furthermore, between-network connectivity (L MCC-R PCC connectivity) $(B=-0.37, P=0.03)$ only uniquely predicted item recognition score $\left[R^{2}=0.26\right.$, $F(3,35)=4.02, P=0.02$; Table S5], not CVLT recall score.

\section{Relationship Between Neuroanatomy, Intrinsic Connectivity, and Memory Performance}

We additionally confirmed that for each network, intrinsic connectivity independently predicted recall memory (CVLT) over and above the anatomical integrity of network regions. DMN neuroanatomy and intrinsic connectivity accounted for $27 \%$ of the variance in CVLT performance $[F(2,35)=6.53, P=0.00$; first data row of Table S6]. SN neuroanatomy and functional connectivity accounted for $26 \%$ of the variance in CVLT performance $[F(2,35)=6.20, P=0.01$; second data row of Table S6]. Then we ran 1 additional regression analysis with anatomical and functional variables of both networks as predictors; we found that they accounted for a total of $44 \%$ of the variance in CVLT performance $[F(4,33)=6.37, P=0.00$; Table $S 7]$. In all cases tested above, the regression models were "not" improved by adding between-network connectivity index (L MCC-R PCC connectivity). Functional connectivity estimates remained significant predictors of memory 1) when we used principal component analysis factor scores as functional connectivity estimates (see statistics highlighted in bold in Table S8) or 2) when we controlled for cortical thickness of seed and target regions that did not differ in thickness between superagers and typical older adults (see statistics highlighted in bold in Tables S9 and S10). Cortical thickness measures did not significantly predict item recognition memory or associative recognition memory so additional regression analyses were not necessary in that regard.

\section{Discussion}

The literature on "successful aging" (Rowe and Kahn 1987; Depp and Jeste 2006; Rowe and Kahn 2015), "cognitive reserve" (Buckner 2004), and "brain maintenance" (Nyberg et al. 2012) points out that age-related cognitive decline is not inevitable. In this study, we add to this growing body of work by showing that superagers exhibit stronger intrinsic connectivity in 2 core intrinsic brain networks-the $\mathrm{DMN}$ and $\mathrm{SN}$-and this connectivity predicted both recall and recognition memory. In addition, we more fully characterized superagers' remarkable memory abilities. Performance on tests of free verbal memory recall, such as the CVLT, is thought to involve largely recollection-based memory processes (Yonelinas 1994; Wixted 2007; Yonelinas et al. 2010; Wolk and Dickerson 2011), as well as controlled search/retrieval processes. In contrast, the ability to correctly identify individual items during a recognition memory test is believed to rely also on familiarity-based memory processes (Yonelinas 1997; Hockley and Consoli 1999; Yonelinas 2002). Our own and others' prior research (Harrison et al. 2012; Sun et al. 2016; Dang et al. 2018; Harrison et al. 2018) demonstrated that superagers have superior free verbal recall performance, suggesting that recollection-based memory processes are superior in them relative to typical older adults. Here we extend this to demonstrate that superagers have youthful item-level recognition memory test performance, suggesting that their familiarity-based memory processes are also better than those of typical older adults. Furthermore, our findings suggest that the association between intrinsic connectivity and memory performance extends generally to both recollection (measured by the CVLT) and familiarity (item recognition memory), and extends across verbal and visual domains. This relationship was strongest within the DMN, although associations between SN connectivity and item recognition memory exhibited similar trends as well. 
Further studies with larger sample sizes are needed to fully characterize the relationship between intrinsic connectivity and recollective versus familiarity-based recognition memory. Overall, these findings suggest that stronger network integrity in superagers provides a process- and domain-general memory advantage.

\section{Intrinsic Functional Integrity as a Neurobiological Substrate for Superaging}

Both the default mode and SNs are implicated in many different psychological and physiological functions (Barrett and Satpute 2013; Yeo et al. 2015; Barrett 2017; Kleckner et al. 2017), including memory function (Buckner et al. 2008; Sestieri et al. 2014). Prior to this study, histologic (Gefen et al. 2015) and morphometric evidence (Harrison et al. 2012; Rogalski et al. 2013; Gefen et al. 2015; Sun et al. 2016; Harrison et al. 2018) provided a static snapshot of superagers' well-maintained neuroanatomy in these networks, which is consistent with a substantial body of literature documenting the relationship between memory and gray matter integrity of key nodes in these 2 networks (e.g., Brickman et al. 2007; Kramer et al. 2007; Head et al. 2008; Sun et al. 2016). Higher gray matter integrity in a region is associated with stronger functional connectivity to connected region (Seeley et al. 2009; Segall et al. 2012; Mutlu et al. 2016), since gray matter atrophy can disrupt synaptic connections to networked regions (network degeneration hypothesis). Given superagers' structural integrity in the DMN and SN, we hypothesized and found they had youthful functional connectivity in the same networks as well. Stronger functional connectivity in superagers was associated with better memory performance, consistent with studies showing that intrinsic connectivity between DMN nodes (e.g., PCC, HF, mPFC) (Andrews-Hanna et al. 2007; Wang et al. 2010; Touroutoglou et al. 2015; Ward et al. 2015; Andreano et al. 2017) and between SN nodes (e.g., AI, pgACC) (AndrewsHanna et al. 2007; Wang et al. 2010; Touroutoglou et al. 2015; Ward et al. 2015; Andreano et al. 2017) predicts memory performance in young adults and in older adults (Lin et al. 2017). We also showed preliminary evidence that superagers had similar level of inverse correlations between DMN and SN nodes as young adults. This is consistent with previous studies showing inverse correlations between intrinsic DMN and SN signals in young adults (Fox et al. 2005; Fransson 2005). As previously reported (He et al. 2014; Keller et al. 2015), this inverse correlation diminishes in typical older adults, but is remarkably youthful in superagers. Importantly, we found that within all older adults, stronger inverse between-network connectivity was associated with better memory performance, consistent with previous findings that between-network connectivity predicts working memory capacity (Keller et al. 2015) and verbal skills (Nashiro et al. 2017). Taken together, these findings suggest that superior memory performance in aging is associated with stronger within-network coupling in the DMN and SN, as well as stronger inverse correlations between these 2 key networks.

We observed that the majority of network targets showed higher bilateral connectivity in superagers, including the AG, dmPFC, rmPFC, pgACC, and sgACC for the DMN (midline clusters in the pgACC and sgACC extended across both hemispheres but had peaks in the right hemisphere), as well as the MCC for the SN. A few targets only showed higher connectivity in the right hemisphere (DMN: aMTG, vlPFC, and HF; SN: SMG), likely because the DMN and SN seeds were both located in the right hemisphere. The SFG was the only target that showed higher connectivity in the left but not right hemisphere, perhaps because the DMN mask used in our study was more restrictive in the right SFG region.

The network seeds (chosen from prior published reports) and targets (identified based on contrast between superagers and typical older adults) that we used in the current study partially overlap with the regions that we previously identified based on neuroanatomy alone (Sun et al. 2016) (Fig. 4). Notably, there were several DMN regions whose cortical thicknesses did not differ between superagers and typical older adults, even though their connectivity profiles did. These include the PCC seed itself, vlPFC, pgACC, and sgACC. On the other hand, there were several $\mathrm{SN}$ regions whose connectivity profiles did not differ between superagers and typical older adults, even as their cortical thicknesses did. These include the MI, dlPFC, and IFG. These findings indicate that reduced cortical thickness or subcortical volume do not necessarily imply reduced intrinsic connectivity (or vice versa), so that both structural and functional variation may

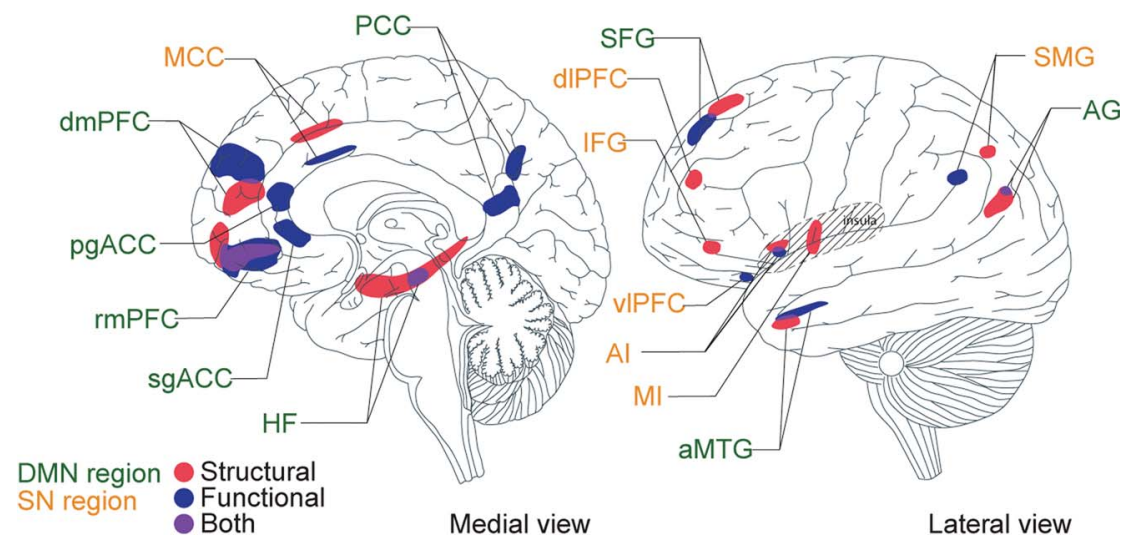

Figure 4. Structural and functional substrates of the superaging brain. Structural substrates (red) were defined based on greater cortical thickness (Sun et al. 2016) and functional substrates (blue) were defined based on stronger intrinsic functional connectivity. Overlapping regions are depicted in purple. Green font indicates DMN regions and orange font indicates SN regions. This is a summary schematic of all substrates combined into 1 hemisphere of the brain, with brain template based on (Tang et al. 2015). 
independently predict unique behavioral variation in memory performance.

\section{Limitations and Future Directions}

It is important to note that the construct of superaging is still evolving and being extended. By definition, superagers are older adults who exhibit episodic memory abilities (RAVLT/CVLT) matching younger counterparts and have average or better processing speed (TMT) for their age. Across different studies, superagers also show age-typical abilities in other cognitive domains such as working memory (n-back) and verbal fluency (category fluency, letter fluency, Boston Naming Test) (Harrison et al. 2012; Sun et al. 2016; Dang et al. 2018).The current study, with a sample size of 17 superagers, was limited in its power to detect small effect sizes. We are planning a future study to recruit a larger sample of superagers. Within the larger context of successful aging (Rowe and Kahn 1987), superaging is only 1 particular model. Previous studies have used a range of behavioral measures such as attention, processing speed, verbal memory, visuospatial memory, visuospatial perception, mental math, and reasoning to define successful aging (Berkman et al. 1993; Inouye et al. 1993; Weintraub et al. 1994; Ylikoski et al. 1999; Andrews et al. 2002; Habib et al. 2007; Whitley et al. 2016; Bott et al. 2017). Other studies also identified high performers (i.e., relative to typical older adults) in memory tasks and examined their brain structure and function (Salami et al. 2014; Lin et al. 2017; Wang et al. 2017; Baran et al. 2018), implicating regions both within and beyond the DMN and SN. It is possible, therefore, that the phenomenon of superaging studied here might also be associated with other behavioral and neural measures, or that superior performance on other measures could represent a different pathway to successful aging. Nonetheless, the present model of superaging does reliably link youthful performance (relative to younger adults) on a well-established verbal recall measure to the integrity of cortical thickness in a specific ensemble of brain regions in the DMN and SN. Future studies should combine multiple cognitive measures into the definition of superaging and perhaps incorporate measures on physical health, lifestyle and social engagement as well. Further, longitudinal studies are needed to understand whether superagers have superior memory performance and greater brain integrity compared with typical older adults because of better preservation or higher baseline. In addition, given that intrinsic connectivity strength in the motor network positively predicted CVLT, future studies could design experiments to examine motor network contributions to superaging. Future studies might more comprehensively characterize functional connectivity for all observable resting-state networks using methods such as the full correlation matrix analysis (Wang et al. 2015). Future research might also investigate neural activation and dynamic connectivity while superagers are performing tasks.

\section{Supplementary Material}

Supplementary material is available at Cerebral Cortex online.

\section{Funding}

National Institutes of Health Director's Pioneer Award (DP1OD003312 to L.F.B.); National Institute on Aging grant (R01 AG030311 to L.F.B. and B.C.D.).

\section{Notes}

The content is solely the responsibility of the authors and does not necessarily represent the official views of the National Institutes of Health or the National Institute on Aging. The authors declare no competing financial interests. Conflict of Interest: None declared.

\section{Author Contributions}

J.M.A., B.C.D., A.T., and L.F.B. designed research; J.Z., J.M.A., B.C.D., A.T., and L.F.B. performed research; J.Z., B.C.D., A.T., and L.F.B. analyzed data; J.Z., B.C.D., A.T., and L.F.B. wrote the paper.

\section{References}

Allen EA, Erhardt EB, Damaraju E, Gruner W, Segall JM, Silva RF, Havlicek M, Rachakonda S, Fries J, Kalyanam R, et al. 2011. A baseline for the multivariate comparison of resting-state networks. Front Syst Neurosci. 5:2.

Andoh J, Matsushita R, Zatorre RJ. 2015. Asymmetric interhemispheric transfer in the auditory network: evidence from TMS, resting-state fMRI, and diffusion imaging. J Neurosci. 35:14602-14611.

Andreano JM, Touroutoglou A, Dickerson BC, Feldman Barrett L. 2017. Resting connectivity between salience nodes predicts recognition memory. Soc Cogn Affect Neurosci. 12:948-955.

Andrews G, Clark M, Luszcz M. 2002. Successful aging in the Australian longitudinal study of aging: applying the MacArthur model cross-nationally. J Soc Issues. 58:749-765.

Andrews-Hanna JR, Snyder AZ, Vincent JL, Lustig C, Head D, Raichle Marcus E, Buckner RL. 2007. Disruption of large-scale brain systems in advanced aging. Neuron. 56:924-935.

Bakkour A, Morris JC, Wolk DA, Dickerson BC. 2013. The effects of aging and Alzheimer's disease on cerebral cortical anatomy: specificity and differential relationships with cognition. Neuroimage. 76:332-344.

Baran TM, Lin FV, Alzheimer's Disease Neuroimaging Initiative. 2018. Amyloid and FDG PET of successful cognitive aging: global and cingulate-specific differences. J Alzheimers Dis. 66:307-318.

Barrett LF. 2017. The theory of constructed emotion: an active inference account of interoception and categorization. Soc Cogn Affect Neurosci. 12:1-23.

Barrett LF, Satpute AB. 2013. Large-scale brain networks in affective and social neuroscience: towards an integrative functional architecture of the brain. Curr Opin Neurobiol. 23:361-372.

Benjamini Y, Hochberg Y. 1995. Controlling the false discovery rate: a practical and powerful approach to multiple testing. J Royal Stat Society Series B Methodol. 57:289-300.

Berkman LF, Seeman TE, Albert M, Blazer D, Kahn R, Mohs R, Finch C, Schneider E, Cotman C, McClearn G et al. 1993. High, usual and impaired functioning in community-dwelling older men and women: findings from the MacArthur Foundation research network on successful aging. J Clin Epidemiol. 46:1129-1140.

Bickart KC, Hollenbeck MC, Barrett LF, Dickerson BC. 2012. Intrinsic amygdala-cortical functional connectivity predicts social network size in humans. J Neurosci. 32:14729-14741.

Biswal B, Yetkin FZ, Haughton VM, Hyde JS. 1995. Functional connectivity in the motor cortex of resting human brain using echo-planar MRI. Magn Reson Med. 34:537-541. 
Bott NT, Bettcher BM, Yokoyama JS, Frazier DT, Wynn M, Karydas A, Yaffe K, Kramer JH. 2017. Youthful processing speed in older adults: genetic, biological, and behavioral predictors of cognitive processing speed trajectories in aging. Front Aging Neurosci. 9:55.

Brickman AM, Habeck C, Zarahn E, Flynn J, Stern Y. 2007. Structural MRI covariance patterns associated with normal aging and neuropsychological functioning. Neurobiol Aging. 28:284-295.

Buckner RL. 2004. Memory and executive function in aging and AD: multiple factors that cause decline and reserve factors that compensate. Neuron. 44:195-208.

Buckner RL, Andrews-Hanna JR, Schacter DL. 2008. The Brain's default network: anatomy, function, and relevance to disease. Ann N Y Acad Sci. 1124:1-38.

Buckner RL, Snyder AZ, Shannon BJ, LaRossa G, Sachs R, Fotenos AF, Sheline YI, Klunk WE, Mathis CA, Morris JC et al. 2005. Molecular, structural, and functional characterization of Alzheimer's disease: evidence for a relationship between default activity, amyloid, and memory. J Neurosci. 25:7709-7717.

Ciric R, Wolf DH, Power JD, Roalf DR, Baum GL, Ruparel K, Shinohara RT, Elliott MA, Eickhoff SB, Davatzikos C et al. 2017. Benchmarking of participant-level confound regression strategies for the control of motion artifact in studies of functional connectivity. Neuroimage. 154:174-187.

Coltheart M. 1981. The MRC psycholinguistic database. Q J Exp Psychol. 33:497-505.

Crivello F, Tzourio-Mazoyer N, Tzourio C, Mazoyer B. 2014. Longitudinal assessment of global and regional rate of grey matter atrophy in 1,172 healthy older adults: modulation by sex and age. PLoS One. 9: e114478.

Dang C, Harrington KD, Lim YY, Ames D, Hassenstab J, Laws SM, Yassi N, Hickey M, Rainey-Smith SR, Robertson J et al. 2018. Superior memory reduces 8-year risk of mild cognitive impairment and dementia but not amyloid beta-associated cognitive decline in older adults. Arch Clin Neuropsychol. https://doi.org/10.1093/arclin/acy078.

Delis DC, Kramer JH, Kaplan E, Ober BA. 1987. California Verbal Learning Test. Research Edition, Manual ed. San Antonio: The Psychological Corporation, Harcourt Brace Jovanovich

Depp CA, Jeste DV. 2006. Definitions and predictors of successful aging: a comprehensive review of larger quantitative studies. Am J Geriatr Psychiatry. 14:6-20.

Dickerson BC, Eichenbaum H. 2009. The episodic memory system: neurocircuitry and disorders. Neuropsychopharmacology. 35:86-104.

Dixon RA, Small BJ, MacDonald SWS, McArdle JJ (2012) Yes, memory declines with aging - but when, how, and why? In: Naveh-Benjamin M, Ohta N, editors. Memory and aging. New York (NY): Taylor \& Francis. p. 325-347.

Esposito F, Aragri A, Pesaresi I, Cirillo S, Tedeschi G, Marciano E, Goebel R, Di Salle F. 2008. Independent component model of the default-mode brain function: combining individual-level and population-level analyses in resting-state fMRI. Magn Reson Imaging. 26:905-913.

Fjell AM, Westlye LT, Grydeland H, Amlien I, Espeseth T, Reinvang I, Raz N, Dale AM, Walhovd KB, Alzheimer Disease Neuroimaging I. 2014. Accelerating cortical thinning: unique to dementia or universal in aging? Cereb Cortex. 24:919-934.

Fox MD, Snyder AZ, Vincent JL, Corbetta M, Van Essen DC, Raichle ME. 2005. The human brain is intrinsically organized into dynamic, anticorrelated functional networks. Proc Natl Acad Sci U S A. 102:9673-9678.

Fransson P. 2005. Spontaneous low-frequency BOLD signal fluctuations: an fMRI investigation of the resting-state default mode of brain function hypothesis. Hum Brain Mapp. 26: 15-29.

Gefen T, Shaw E, Whitney K, Martersteck A, Stratton J, Rademaker A, Weintraub S, Mesulam MM, Rogalski E. 2014. Longitudinal neuropsychological performance of cognitive superagers. J Am Geriatr Soc. 62:1598-1600.

Gefen T, Peterson M, Papastefan ST, Martersteck A, Whitney K, Rademaker A, Bigio EH, Weintraub S, Rogalski E, Mesulam MM et al. 2015. Morphometric and histologic substrates of cingulate integrity in elders with exceptional memory capacity. J Neurosci. 35:1781-1791.

Grady CL, Craik FI. 2000. Changes in memory processing with age. Curr Opin Neurobiol. 10:224-231.

Grady CL, Grigg O, Ng C. 2012. Age differences in default and reward networks during processing of personally relevant information. Neuropsychologia. 50:1682-1697.

Greicius MD, Kimmel DL. 2012. Neuroimaging insights into network-based neurodegeneration. Curr Opin Neurol. 25:727-734.

Greicius MD, Krasnow B, Reiss AL, Menon V. 2003. Functional connectivity in the resting brain: a network analysis of the default mode hypothesis. Proc Natl Acad Sci U S A. 100:253-258.

Habib R, Nyberg L, Nilsson L-G. 2007. Cognitive and non-cognitive factors contributing to the longitudinal identification of successful older adults in the Betula study. Aging Neuropsychol Cogn. 14:257-273.

Harrison TM, Weintraub S, Mesulam MM, Rogalski E. 2012. Superior memory and higher cortical volumes in unusually successful cognitive aging. J Int Neuropsychol Soc. 18:1081-1085.

Harrison TM, Maass A, Baker SL, Jagust WJ. 2018. Brain morphology, cognition, and $\beta$-amyloid in older adults with superior memory performance. Neurobiol Aging. 67:162-170.

He X, Qin W, Liu Y, Zhang X, Duan Y, Song J, Li K, Jiang T, Yu C. 2013. Age-related decrease in functional connectivity of the right fronto-insular cortex with the central executive and default-mode networks in adults from young to middle age. Neurosci Lett. 544:74-79.

He X, Qin W, Liu Y, Zhang X, Duan Y, Song J, Li K, Jiang T, Yu C. 2014. Abnormal salience network in normal aging and in amnestic mild cognitive impairment and Alzheimer's disease. Hum Brain Mapp. 35:3446-3464.

Head D, Rodrigue KM, Kennedy KM, Raz N. 2008. Neuroanatomical and cognitive mediators of age-related differences in episodic memory. Neuropsychology. 22:491-507.

Hedden T, Gabrieli JD. 2004. Insights into the ageing mind: a view from cognitive neuroscience. Nat Rev Neurosci. 5:87-96.

Herlitz A, Rehnman J. 2008. Sex differences in episodic memory. Curr Dir Psychol Sci. 17.

Herlitz A, Nilsson LG, Backman L. 1997. Gender differences in episodic memory. Mem Cognit. 25:801-811.

Hockley WE, Consoli A. 1999. Familiarity and recollection in item and associative recognition. Mem Cognit. 27:657-664.

Inouye SK, Albert MS, Mohs R, Sun K, Berkman LF. 1993. Cognitive performance in a high-functioning community-dwelling elderly population. J Gerontol. 48:M146-M151.

Keller JB, Hedden T, Thompson TW, Anteraper SA, Gabrieli JD, Whitfield-Gabrieli S. 2015. Resting-state anticorrelations between medial and lateral prefrontal cortex: association 
with working memory, aging, and individual differences. Cortex. 64:271-280.

Kim H. 2010. Dissociating the roles of the default-mode, dorsal, and ventral networks in episodic memory retrieval. Neuroimage. 50:1648-1657.

Kleckner IR, Zhang J, Touroutoglou A, Chanes L, Xia C, Simmons WK, Quigley KS, Dickerson BC, Barrett LF. 2017. Evidence for a large-scale brain system supporting allostasis and interoception in humans. Nat Hum Behav. 1.

Konishi S, Wheeler ME, Donaldson DI, Buckner RL. 2000. Neural correlates of episodic retrieval success. Neuroimage. 12:276-286.

Kramer JH, Mungas D, Reed BR, Wetzel ME, Burnett MM, Miller BL, Weiner MW, Chui HC. 2007. Longitudinal MRI and cognitive change in healthy elderly. Neuropsychology. 21: $412-418$.

Lang PJ, Bradley MM, Cuthbert BN (1997) International Affective Picture System (IAPS): Technical Manual and Affective Ratings. Gainesville, FL: NIMH Center for the Study of Emotion and Attention.

Langner R, Cieslik EC, Behrwind SD, Roski C, Caspers S, Amunts K, Eickhoff SB. 2015. Aging and response conflict solution: behavioural and functional connectivity changes. Brain Struct Funct. 220:1739-1757.

Lepage M, Ghaffar O, Nyberg L, Tulving E. 2000. Prefrontal cortex and episodic memory retrieval mode. Proc Natl Acad Sci U S A. 97:506-511.

Lin F, Ren P, Mapstone M, Meyers SP, Porsteinsson A, Baran TM. 2017. The cingulate cortex of older adults with excellent memory capacity. Cortex. 86:83-92.

McGinnis SM, Brickhouse M, Pascual B, Dickerson BC. 2011. Agerelated changes in the thickness of cortical zones in humans. Brain Topogr. 24:279-291.

Menon V (2015) Salience network. In: Toga AW, editor. Brain mapping: an encyclopedic reference. Oxford, UK: Elsevier. p. 597-611.

Menon V, Uddin LQ. 2010. Saliency, switching, attention and control: a network model of insula function. Brain Struct Funct. 214:655-667.

Minear M, Park DC. 2004. A lifespan database of adult facial stimuli. Behav Res Methods Instrum Comput. 36:630-633.

Mutlu J, Landeau B, Tomadesso C, de Flores R, Mezenge F, de La Sayette V, Eustache F, Chetelat G. 2016. Connectivity disruption, atrophy, and hypometabolism within posterior cingulate networks in Alzheimer's Disease. Front Neurosci. 10:582.

Nashiro K, Sakaki M, Braskie MN, Mather M. 2017. Resting-state networks associated with cognitive processing show more age-related decline than those associated with emotional processing. Neurobiol Aging. 54:152-162.

Nyberg L, Lovden M, Riklund K, Lindenberger U, Backman L. 2012. Memory aging and brain maintenance. Trends Cogn Sci. 16:292-305.

Onoda K, Ishihara M, Yamaguchi S. 2012. Decreased functional connectivity by aging is associated with cognitive decline. J Cogn Neurosci. 24:2186-2198.

Palop JJ, Chin J, Mucke L. 2006. A network dysfunction perspective on neurodegenerative diseases. Nature. 443:768-773.

Park DC, Reuter-Lorenz P. 2009. The adaptive brain: aging and neurocognitive scaffolding. Annu Rev Psychol. 60:173-196.

Raichle ME, MacLeod AM, Snyder AZ, Powers WJ, Gusnard DA, Shulman GL. 2001. A default mode of brain function. Proc Natl Acad Sci U S A. 98:676-682.
Rogalski EJ, Gefen T, Shi J, Samimi M, Bigio E, Weintraub S, Geula C, Mesulam MM. 2013. Youthful memory capacity in old brains: anatomic and genetic clues from the Northwestern SuperAging project. J Cogn Neurosci. 25:29-36.

Roski C, Caspers S, Langner R, Laird AR, Fox PT, Zilles K, Amunts K, Eickhoff SB. 2013. Adult age-dependent differences in resting-state connectivity within and between visual-attention and sensorimotor networks. Front Aging Neurosci. 5:67.

Rowe JW, Kahn RL. 1987. Human aging: usual and successful. Science. 237:143-149.

Rowe JW, Kahn RL. 2015. Successful aging 2.0: conceptual expansions for the 21st century. J Gerontol B Psychol Sci Soc Sci. 70:593-596.

Salami A, Pudas S, Nyberg L. 2014. Elevated hippocampal resting-state connectivity underlies deficient neurocognitive function in aging. Proc Natl Acad Sci U S A. 111: 17654-17659.

Seeley WW, Crawford RK, Zhou J, Miller BL, Greicius MD. 2009. Neurodegenerative diseases target large-scale human brain networks. Neuron. 62:42-52.

Seeley WW, Menon V, Schatzberg AF, Keller J, Glover GH, Kenna H, Reiss AL, Greicius MD. 2007. Dissociable intrinsic connectivity networks for salience processing and executive control. J Neurosci. 27:2349-2356.

Segall JM, Allen EA, Jung RE, Erhardt EB, Arja SK, Kiehl K, Calhoun VD. 2012. Correspondence between structure and function in the human brain at rest. Front Neuroinform. 6:10.

Sestieri C, Corbetta M, Spadone S, Romani GL, Shulman GL. 2014. Domain-general signals in the cingulo-opercular network for visuospatial attention and episodic memory. J Cogn Neurosci. 26:551-568.

Squire LR, Stark CEL, Clark RE. 2004. The medial temporal lobe. Annu Rev Neurosci. 27:279-306.

Sun FW, Stepanovic MR, Andreano J, Barrett LF, Touroutoglou A, Dickerson BC. 2016. Youthful brains in older adults: preserved neuroanatomy in the default mode and salience networks contributes to youthful memory in superaging. J Neurosci. 36:9659-9668.

Tang YY, Holzel BK, Posner MI. 2015. The neuroscience of mindfulness meditation. Nat Rev Neurosci. 16:213-225.

Tombaugh TN. 2004. Trail Making Test A and B: normative data stratified by age and education. Arch Clin Neuropsychol. 19:203-214.

Touroutoglou A, Hollenbeck M, Dickerson BC, Feldman Barrett L. 2012. Dissociable large-scale networks anchored in the right anterior insula subserve affective experience and attention. Neuroimage. 60:1947-1958.

Touroutoglou A, Bickart KC, Barrett LF, Dickerson BC. 2014. Amygdala task-evoked activity and task-free connectivity independently contribute to feelings of arousal. Hum Brain Mapp. 35:5316-5327.

Touroutoglou A, Andreano JM, Barrett LF, Dickerson BC. 2015. Brain network connectivity-behavioral relationships exhibit trait-like properties: evidence from hippocampal connectivity and memory. Hippocampus. 25:1591-1598.

Touroutoglou A, Zhang J, Andreano JM, Dickerson BC, Barrett LF. 2018. Dissociable effects of aging on salience subnetwork connectivity mediate age-related changes in executive function and affect. Front Aging Neurosci. 10:410.

Touroutoglou A, Bliss-Moreau E, Zhang J, Mantini D, Vanduffel W, Dickerson BC, Barrett LF. 2016. A ventral salience network in the macaque brain. Neuroimage. 132:190-197. 
Van Dijk KR, Sabuncu MR, Buckner RL. 2012. The influence of head motion on intrinsic functional connectivity MRI. Neuroimage. 59:431-438.

van Dijk KR, Hedden T, Venkataraman A, Evans KC, Lazar SW, Buckner RL. 2010. Intrinsic functional connectivity as a tool for human connectomics: theory, properties, and optimization. J Neurophysiol. 103:297-321.

Vincent JL, Kahn I, Snyder AZ, Raichle ME, Buckner RL. 2008. Evidence for a frontoparietal control system revealed by intrinsic functional connectivity. J Neurophysiol. 100:3328-3342.

Vincent JL, Patel GH, Fox MD, Snyder AZ, Baker JT, Van Essen DC, Zempel JM, Snyder LH, Corbetta M, Raichle ME. 2007. Intrinsic functional architecture in the anaesthetized monkey brain. Nature. 447:83-86.

Wang L, Su L, Shen H, Hu D. 2012. Decoding lifespan changes of the human brain using resting-state functional connectivity MRI. PLoS One. 7: e44530.

Wang L, LaViolette P, O'Keefe K, Putcha D, Bakkour A, Van Dijk KRA, Pihlajamäki M, Dickerson BC, Sperling RA. 2010. Intrinsic connectivity between the hippocampus and posteromedial cortex predicts memory performance in cognitively intact older individuals. Neuroimage. 51:910-917.

Wang X, Ren P, Baran TM, Raizada RDS, Mapstone M, Lin F, Alzheimer's Disease Neuroimaging I. 2017. Longitudinal functional brain mapping in supernormals. Cereb Cortex. 29:1-11.

Wang Y, Cohen JD, Li K, Turk-Browne NB. 2015. Full correlation matrix analysis (FCMA): an unbiased method for task-related functional connectivity. J Neurosci Methods. 251:108-119.

Ward AM, Mormino EC, Huijbers W, Schultz AP, Hedden T, Sperling RA. 2015. Relationships between default-mode network connectivity, medial temporal lobe structure, and agerelated memory deficits. Neurobiol Aging. 36:265-272.
Weintraub S, Powell DH, Whitla DK. 1994. Successful cognitive aging: individual differences among physicians on a computerized test of mental state. J Geriatr Psychiatry. 28:15-34.

Whitley E, Popham F, Benzeval M. 2016. Comparison of the RoweKahn model of successful aging with self-rated health and life satisfaction: the west of Scotland Twenty-07 prospective cohort study. Gerontologist. 56:1082-1092.

Wiggs CL, Weisberg J, Martin A. 1999. Neural correlates of semantic and episodic memory retrieval. Neuropsychologia. 37:103-118.

Wixted JT. 2007. Dual-process theory and signal-detection theory of recognition memory. Psychol Rev. 114:152-176.

Wolk DA, Dickerson BC. 2011. Fractionating verbal episodic memory in Alzheimer's disease. Neuroimage. 54:1530-1539.

Yeo BT, Krienen FM, Eickhoff SB, Yaakub SN, Fox PT, Buckner RL, Asplund CL, Chee MW. 2015. Functional specialization and flexibility in human association cortex. Cereb Cortex. 25:3654-3672.

Ylikoski R, Ylikoski A, Keskivaara P, Tilvis R, Sulkava R, Erkinjuntti T. 1999. Heterogeneity of cognitive profiles in aging: successful aging, normal aging, and individuals at risk for cognitive decline. Eur J Neurol. 6:645-652.

Yonelinas AP. 1994. Receiver-operating characteristics in recognition memory: evidence for a dual-process model. J Exp Psychol Learn Mem Cogn. 20:1341-1354.

Yonelinas AP. 1997. Recognition memory ROCs for item and associative information: the contribution of recollection and familiarity. Mem Cognit. 25:747-763.

Yonelinas AP. 2002. The nature of recollection and familiarity: a review of 30 years of research. J Mem Lang. 46:441-517.

Yonelinas AP, Aly M, Wang WC, Koen JD. 2010. Recollection and familiarity: examining controversial assumptions and new directions. Hippocampus. 20:1178-1194. 